\title{
DIVISION IV / WORKING GROUP on MASSIVE STARS
}

CHAIR
VICE-CHAIR
PAST CHAIR
BOARD

\author{
Joachim Puls \\ Claus Leitherer \\ Stan Owocki \\ Paul Crowther \\ Margaret Hanson \\ Artemio Herrero \\ Norbert Langer \\ Stan Owocki \\ Gregor Rauw \\ Nicole St-Louis \\ Richard Townsend
}

\section{TRIENNIAL REPORT 2010-2012}

\section{Introduction and background}

Our Working Group (WG) studies massive, luminous stars, both individually and in resolved and unresolved populations, with historical focus on early-type (OB) stars, Asupergiants, and Wolf-Rayet stars. Our group also studies lower mass stars (e.g., central stars of planetary nebulae and their winds) which display features similar or related to those present in massive stars, and thus may improve our understanding of the physical processes occurring in massive stars. In recent years, massive red supergiants that evolve from hot stars have been included into our activities as well. We emphasize the role of massive stars in other branches of astrophysics, particularly regarding the First Stars, long duration Gamma-Ray bursts, formation of massive stars and their feedback on star formation in general, pulsations of massive stars, and starburst galaxies.

The goal of this Working Group is to focus research in the massive star community and to help communicating the most recent results. Minor and major conferences shall be proposed, (co-)sponsored, and/or help in the application process shall be provided. Most prominent are the 'massive star conferences' covering major topics in massive star research, held about every five years, with many of them under the auspices of the International Astronomical Union.

By-laws generated and approved by the Organization Committee (OC) and other activities of the Working Group are posted on an offical website at:

http://www.astroscu. unam.mx/massive_stars

The webmaster for this site is Raphael Hirschi (University of Keele, UK).

\section{Developments within the past triennium}

Over the past two years, and projected to 2012, WG activities focussed on issues related to the "Massive Star Newletter", and on co-sponsorship and preparation of various conferences/workshops. In particular, the OC called for proposals regarding Joint Discussions (JD) and Special Sessions (SpS) to be held during the IAU GA 2012 in Beijing, 
and helped with the preparation of the corresponding events. The follow gives further details.

\subsection{The Massive Star Newletter}

Among other features, our web portal offers an automatic abstract submission interface. This allows the members of the Working Group to submit recent abstracts and circulates newly received abstracts to registered members, both individually as well as collected within The Massive Star Newsletter (together with other topics of interest such as finished thesis work, upcoming conferences, and job offers).

The Newsletter, edited by Philippe Eenens (Universidad de Guanajuato, Mexico) and Raphael Hirschi, continues to be the main means of communication and science propagation in our Working Group. As of October 2011, 124 issues of the Newsletter have been published. Back issues are posted on the Working Group website:

http: //www . astroscu. unam.mx/massive_stars/news.php

During the past two years, the WG has helped Raphael Hirschi and Philippe Eeenens in switching from a 'manual' production of the Newsletter to an automatic process.

\subsection{Workshop - Hot and Cool: Bridging Gaps in Massive Star Evolution $\dagger$}

Held November 10-12, 2008, on the campus of the California Institute of Technology, and hosted by the NASA Herschel Science Center.

The topic of this workshop was a result of intense (and still ongoing!) discussions within the WG to bridge the gap between and bring together researchers studying stars in the upper blue and red parts of the Hertzsprung-Russell diagram (HRD). While morphologically separated, stars occupying these extremes of the HRD are intimately related via evolution, as well as both having atmospheric properties affected by extension and mass outflow. At cosmological scales, like in distant starburst galaxies, the historical distinction between blue and red stellar populations becomes obsolete, and understanding the complex relation between the red and blue parts of the HRD is mandatory.

The meeting, chaired by Claus Leitherer, member of our OC, was intended to investigate similarities and differences between hot and cool stars, identify the most challenging questions in evolutionary connections, and discuss latest theoretical and observational progress, to provide a more unified understanding of massive stars and luminous extragalactic stellar populations. Major topics included:

- Evolutionary connections

- Late and end phases (post-RSG and blue-loop stars, supernovae from red vs. blue progenitors, Gamma-ray bursts)

- Atmospheres

- Mass loss (rates, physics, episodic mass loss, pulsations)

- Interacting winds and circumstellar environments

Further information can be found in the workshop proceedings edited by Leitherer et al. (2010).

\subsection{JD2 at IAU GA 2012 - 'Very Massive Stars in the Local Universe'}

This 1.5 day JD, to be held during the IAU GA 2012 in Beijing, is co-sponsored by our WG, and will be chaired by Jorick Vink (Armagh, Ireland).

$\dagger$ Technically, this workshop falls outside the reporting period, but was not covered in the last report, because being held at the end of 2008 . 
Recent findings indicating the existence of very massive stars (VMS) up to $\sim 300 \mathrm{M}_{\odot}$ in the local Universe (Gal-Yam et al. 2009, Crowther et al. 2010) might lead to a paradigm shift for the stellar upper-mass limit. The JD intends to discuss the status of the data, as well as the far-reaching implications of such objects.

The determination of both current and final masses of the most massive stars shall be discussed, to reach consensus between observers and theorists on how to identify and quantify the importance of the dominant physical processes. The objects may evolve almost chemically homogeneously, implying that the detailed mixing processes (e.g., rotation, magnetic fields) could be less relevant compared to $10-50 \mathrm{M}_{\odot}$ stars. Instead, the evolution and death of VMS is likely dominated by mass loss. Major topics of this JD include

- Weighing the most massive stars from their binary motions

- Mass determinations from stellar spectroscopy and model atmosphere analysis

- Formation of the most massive stars

- Mass loss mechanisms, incl. eruptions of Luminous Blue Variables

- Stellar structure and evolution modeling

- The fate of the most massive stars (over cosmological time)

- Mass and energy return to the interstellar medium (ISM)

Further information at the website http://www.arm.ac.uk/IAU/index.html

\subsection{SpS5 at IAU GA 2012 - 'The IR view of massive stars: the main sequence and beyond'}

This 1.5 day SpS, to be held during the IAU GA 2012 in Beijing, is co-sponsored by our WG, and will be chaired by Yaël Nazé (Liège, Belgium).

Even though a major fraction of astronomers still relies on the optical domain, this is certainly going to change, as most current and future instruments are dedicated to the infrared, from the near- to the far-IR bands. While this domain is a known 'must' for (very) low-mass stars, the infrared emission of high-mass stars has been often neglected. Many advantages of the infrared need to be stressed, however, like its strong potential for circumstellar material and atmosphere diagnostics, and its insensitivity to obscuration. Its interest with respect to the first generation of stars is also well known.

In this $\mathrm{SpS}$, it is intended to discuss the results obtained for massive stars from existing IR facilities (VLTs/VLTI, Spitzer, Herschel, CRIRES, GAIA, ...) as well as tools for interpreting IR data (e.g. atmosphere modeling) and observing capabilities of future facilities (ELTs, JWST, ...). Major topics to be discussed are

- Obscured and distant clusters in the IR

- Stellar and wind parameters from the IR

- Matter ejection and feedback

Further information at the website

http: //www.gaphe.ulg.ac.be/IAU_XXVIII/index.html

\section{Future outlook}

Our WG is presently involved in the preparation of the upcoming 'massive star conference', planned to be held in 2013 in Rhodes (Greece), with the title "Massive Stars: from alpha to Omega".

Other upcoming meetings on massive stars include: 
- "Circumstellar Dynamics at High Resolution", to be held February 27 - March 2, 2012, in Foz do Iguaçu, Brazil.

Weblink: http://www.eso.org/sci/meetings/2012/csdyn.html

- "The Evolution of Massive Stars and Progenitors of GRBs", to be held June 17 - July 1, 2012, at the Aspen Center for Physics, Aspen, CO. Further details at

http: //casa.colorado.edu/ emle6425/aspen/

In the recent few years, but even more in the near future, exciting new results on massive stars have been and will be obtained by spectropolarimetry, interferometry and asteroseismology. These techniques probe both the physics of the surfaces and interiors of massive stars, allowing unprecedented constraints of key physical parameters such as rotation, magnetic fields, and chemical composition.

Large-scale, high-quality surveys of resolved stellar populations (e.g the VLT-FLAMES survey on massive stars and the Tarantula survey), as well as highly detailed investigations of individual systems (e.g. $\eta$ Car), have exposed key weaknesses in our assumptions on massive-star evolution. It has become clear now that understanding stellar populations is a considerable challenge that will require substantial efforts to resolve. We envisage interesting times for the massive star community.

\section{Acknowledgements}

The OC gratefully acknowledges logistic support for several teleconferences by STScI. Many thanks to the Institute of Astronomy of UNAM (Mexico) for support with their web server.

Joachim Puls chair of Working Group

\section{References}

Crowther, P. A., Schnurr, O., Hirschi, R., et al. 2010, MNRAS, 408, 731

Gal-Yam, A., Mazzali, P., Ofek, E. O., et al. 2009, Nature, 462, 624

Leitherer, C., Bennett, P., Morris, P., \& van Loon, J. (editors) 2010, Astronomical Society of the Pacific Conference Series, 425 